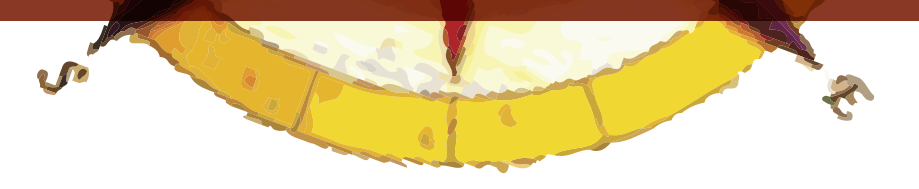

\title{
LA DIDÁCTICA DE LA GEOGRAFÍA DENTRO DE UN CAMPO CONCEPTUAL DE LA PEDAGOGÍA: TENSIONES Y BIFURCACIONES.
}

\author{
María Alejandra Taborda Caro ${ }^{1}$
}

\section{RESUMEN}

Desde diferentes lugares y posiciones se acepta hoy, que la didáctica de la geografia transita por una fuerte crisis en relación con la enseñanza; crisis en el qué, en el cómo y en el para qué, y sufre una pérdida de identidad. Hoy la didáctica de la geografía como un campo de reflexión propio e independiente integrado a las prácticas educativas está mediado por la posibilidad de integrarse a campos de problemáticas, de discusiones amplias con otras didácticas específicas, con la didáctica general y con el amplio espectro de las ciencias sociales y su didáctica. Adicionalmente la didáctica de la geografía requiere sortear vastas discusiones sobre las relaciones que mantiene con la pedagogía y la educación. Debates todos estos, de una enorme complejidad y ambigüedad especialmente en momentos en que el campo de lo social pareciera adsorber la existencia misma de lo geográfico.

En medio de estas discusiones no resueltas y en muchos casos no iniciadas, resulta la geografía pensada por la historia de las disciplinas escolares por un lado y por otro integrada a la didáctica de las ciencias sociales. Este artículo intentará aproximarse al estado de esta discusión, y las tensiones que generan estas fuerzas dentro del campo de la pedagogía.

Palabras clave: didáctica, geografía, campo, ciencias sociales

\begin{abstract}
From different places and positions is now accepted that the teaching of geography is undergoing a severe crisis in relation to education, crisis in the "what", the "how " and the "why " and suffers a loss of identity. today the teaching of geography as a field of its own independent reflection integrated educational practices is mediated by the ability to integrate a problematic fields, extensive discussions with other specific teaching with general teaching and the broad spectrum of social Science and Teaching . Additionally, the teaching of geography requires circumvent extensive discussions on relations with maintaining the pedagogy and education. Debates of all these enormous
\end{abstract}

Docente de la Universidad de Córdoba-Colombia. 
complexity, ambiguity and especially at a time when the social field seems adsorb the existence of geographically .

Amid these unresolved discussions and in many cases not initiated, it is thought by geography history of school subjects on the one hand and on the other integrated into the teaching of the social sciences. This article tried to approach the status of this discussion, and the tensions generated by these forces within the field of education

Keywords: teaching, geography, field, social sciences.

\section{RESUMO}

Com diferentes localizações e posições é agora aceite que o ensino de geografia está passando por uma crise grave em relação à educação, crise no "o quê", o "como" eo "porquê" e sofre uma perda de identidade . hoje o ensino da geografia como um campo de suas próprias práticas educativas integradas reflexão independentes é mediada pela capacidade de integrar um campos problemáticos, amplas discussões com outro ensinamento específico com o ensino geral eo amplo espectro de Ciências Sociais e Ensino . Além disso, o ensino da geografia requer contornar amplos debates sobre as relações com a manutenção da pedagogia e da educação. Debates de todos estes enorme complexidade, ambigüidade e especialmente num momento em que o campo social parece absorver a existência de geograficamente.

Em meio a essas discussões não resolvidas e em muitos casos não iniciadas , pensa-se pela história geografia das disciplinas escolares, por um lado e , por outro integrado ao ensino das ciências sociais. Este artigo tentou se aproximar do estatuto desta discussão, e as tensões geradas por essas forças dentro do campo da educação.

Palavras-chave: ensino, geografia, país, ciências sociais

\section{LA DINÁMICA DE LA GEOGRAFÍA ESCOLAR DENTRO DE UN CAMPO CONCEPTUAL DE LA PEDAGOGIA.}

Una lectura a el lugar que configura hoy la didáctica de la geografía como un campo de reflexión propio e independiente integrado a las prácticas educativas está mediado por la posibilidad de integrarse a campos de problemáticas, de discusiones amplias con otras didácticas específicas, con la didáctica general y con el amplio espectro de las ciencias sociales y su didáctica. Adicionalmente la didáctica de la geografía requiere sortear amplias discusiones sobre las relaciones que mantiene la con la pedagogía y la educación. Debates todos estos de una enorme complejidad, y ambigüedad especialmente en momentos en que el campo de lo social pareciera adsorber la existencia misma de lo geográfico.

Así mismo mediados del siglo pasado una enorme producción de textos e investigaciones referidas a la didáctica de la geografía se difundieron la mayor parte de ellos preocupados por la manera 
como los estudiantes se integraban al medio a través de sus propias experiencias, (en ese sentido el trabajo de campo era fundamental). En la década siguiente, una diversidad de autores consideró la didáctica de la geografía colocando un gran énfasis en la necesidad de conocer "cómo aprenden los alumnos", y más concretamente sus dificultades de aprendizaje; predominaron los estudios referidos a la formación de conceptos, más adelante la preocupación se desvió hacia lo relativo a la enseñanza con las nuevas tecnologías.

Este proceso de común ha tenido para la formación docente una preocupación por instaurar nuevos contenidos geográficos, y la poca reflexión al carácter teórico y conceptual, sobre lo que podría ser la didáctica de la geografía como campo de estudio, y sus relaciones con las didácticas generales.

Lo anterior le ha exigido a la didáctica de la geografía su integración a un campo conceptual de la pedagogía ${ }^{2}$ - CCP - que para Echeverri (2010) es un espacio creado para el ejercicio discursivo en el cual un grupo o grupos de intelectuales conceptúan, modifican o cambian ideologías, teorías y prácticas en el proceso de producción del discurso educativo dentro de un escenario de luchas internas, en el que se generan espacios para las convenios simbólicos entre grupos de intelectuales, que se disponen en tensión permanente por asumir posiciones discursivas y hegemónicas. Por consiguiente, la didáctica de la geografía no se configura hoy como una disciplina aislada, con problemas propios; muy por el contrario su existencia está condicionada a la capacidad que tenga de dialogar, negociar y de dar respuestas a problemas comunes con un andamiaje conceptual distintivo.

Aclaramos que la noción de campo conceptual, que aquí se adopta no tiene sus raíces en preocupaciones por el estatuto epistemológico ni de la enseñanza de la geografía, ni de la geografía, sino en la mirada de la didáctica desde la concepción de saber $^{3}$ que vincula a la didáctica de la geografía con una vieja y actual tradición de las teorías pedagógicas con sus conceptos, problemas y sus prácticas reafirmadas a través de la escuela y su historia. El saber pedagógico y didáctico surge de la reflexión sistemática sobre la práctica, tal como lo describe Tezanos (2007):

El saber sólo puede ser producido en el espacio y tiempo real en el cual los profesores preparan sus lecciones, las desarrollan, valoran el trabajo y los aprendizajes de sus alumnos, toman en cuenta el contexto en el cual llevan a cabo su quehacer docente, deciden y emplean materiales didácticos de acuerdo a los contenidos que enseñan y las condiciones y características de sus estudiantes. Estos elementos, y fundamentalmente las relaciones entre ellos, están en el origen de la construcción de saber pedagógico (p. 22).

De esta manera la categoría de saber pedagógico le permite a didáctica de la geografia una arti-

2 La noción de campo conceptual de la pedagogía - noción es construida en el seno del trabajo colectivo del grupo de investigación ACIFORMA liderado por el profesor Alberto Echeverri. La conceptualización sobre la pedagogía en Colombia entre 1970 y el 2000, es el demostrar que ésta tiene la potencia suficiente para constituirse en un CCP, a diferencia de los enfoques que no ven en la pedagogía la fuerza para transformarse en un campo de producción conceptual. Este aporte se personifica en las siguientes preguntas: ¿Es la pedagogía una simple producción ideológica entre otras, o un simple reflejo de las estructuras económicas, políticas, culturales o simbólicas?

3 La construcción de saber es una expresión ligada a los trabajos de Foucault, que durante los años ochenta emerge en la discusión como un concepto clave para dar cuenta de aquello que está fuera de los modos de operar de lo científico, sin que esto implique una condición de inferioridad o superioridad con respecto de aquello, sino que establece distinciones fructíferas y clarificadoras en las argumentaciones, fundamentalmente aquellas ligadas a la constitución de la idea de profesión. Y es desde una lectura rigurosa y creativa que Olga Lucía Zuluaga, en su texto Historia y Pedagogía, define las condiciones de posibilidad del saber pedagógico. 
culación con CCP a través de un diálogo interdisciplinar que facilita un compartir, platicar, discutir, analizar entre pares y opuestos, al respecto nos recuerda Garcés (2007), a propósito de las tensiones sobre su legitimidad que la didáctica de la geografía enfrenta hoy, que sólo cuando, una estructura conceptual es sometida al juicio riguroso, objetivo y sistemático para trascender la mera opinión de los colegas, se puede afirmar que se está construyendo saber pedagógico. Por otro lado, la ciencia desde otra óptica reclama respuestas que analicen, expliquen e interpreten los fenómenos naturales y sociales que rodean al ser humano.

Conviene aclarar aquí que si proponemos mirar la didáctica de la geografía desde la noción de campo conceptual de la pedagogía es porque nos despojamos de las variadas clasificaciones derivadas de las varias propuestas de carácter epistemológico; clasificaciones que ponen a la pedagogía en una situación precaria frente a otros saberes que pretenden hegemonizar el discurso pedagógico. Con todo y lo anterior nos preocupa entonces rescatar para la didácticas reflexiones pedagógicas en su tradición y su conceptualidad y problematizar la marcada psicología constructivista y sociología educativa que han trazado la reflexión de la didáctica de la geografía desde mediados del siglo pasado.

Descrito así el campo, y sus implicaciones para la didáctica de la geografía, éste nos permite describir y mostrar las principales tensiones que se enfrentan cuando se intenta legitimar un discurso sobre la enseñanza de la geografía; surgen algunas preguntas a resolver ¿la didáctica de la geografia es un subcampo de la ¿didáctica de las ciencias sociales? ¿tiene un cuerpo teórico y unas aporte metodológicos propios? ¿cuáles son las dinámicas de dependencia o de autonomía con la psicología y la sociología educativa? ¿Cómo estable sus diálogos con su disciplina referente la geografia?, algunos de los derroteros a seguir para establecer puntos de confluencia de la didáctica de la geografia con el amplio espectro de la pedagogía y la didáctica deberá contemplar algunos aspectos a saber. Entre ellos, es pertinente considerar:

El tratamiento de problemas del CCP incluyendo la historicidad de los conceptos y problemas que a través del tiempo fueron configurando unas formas, metodologías, técnicas y unos discursos propios de la enseñanza de la geografia es decir se requiere reconocer unas especificidades y unas apropiaciones.

Que en la actualidad la identidad de la didáctica de la geografia es muy débil, y que para algunos aún no se define bajo una forma de disciplina sino bajo la forma de práctica y de discurso híbrido en el que se genera una yuxtaposición de disciplinas tales como la psicología (cognoscitivismo, humanismo, piagetismo, vigotskismo, conductismo etc.). Las ciencias sociales (sociología, historia, antropología), la didáctica de las ciencias sociales y de la historia, y más recientemente la ciudadanía, requieren legitimar posiciones frente a la consideración de los temas didácticos referidos a la geografia y configurados como una región de saber.

Los discursos multidisciplinarios pues éstos constituyen las disciplinas como campos estratégicos donde las posiciones, oposiciones y disposiciones en torno al discurso pedagógico y didáctico estructuran un campo; si consideramos que los campos son esencialmente estratégicos, es decir, se estructuran a partir de las relaciones de fuerzas entre los 'agentes' o 'las instituciones' implicadas en la lucha, relaciones de fuerza que determinan la distribución del 'capital específico acumulado' en el curso de las luchas anteriores y la cual orienta las estrategias futuras. 
Se hace necesario renovar los contenidos, reorientándolos hacia una aproximación explicativa, demodo tal que permitan comprender la realidad actual a distintas escalas. Hoy, como vimos, corremos con la ventaja que el marco institucional lo permite; en consecuencia se hace necesario revisar las formas, el "cómo" enseñar. Debemos abordar tanto la actualización de contenidos como la de los instrumentos. A veces se observa que los fracasos obedecen más a la falta de habilidades y/o actitudes adecuadas que al dominio de conocimientos.

Se concluye que al reivindicar la existencia de un campo conceptual de la pedagogía, se insiste en dos aspectos: por un lado, la pluralidad y el estado de apertura en el campo, y por otro lado, en la historicidad del campo y de los conceptos que los conforman. En otras palabras, el campo conceptual de la pedagogía no responde a criterios metodológicos o discursivos ajenos sino que está atento a la tradición misma de la pedagogía, a su peculiar historia. Zuluaga no teme atribuirle a la historia de conceptos la tarea de proporcionar los recursos metodológicos para acceder a la historia de la pedagogía y lo ganado hasta ahora en el presente trabajo mediante la, Garcés (2007) tomado de Echeverri y Zuluaga.

\section{LA DIDÁCTICA DE LA GEOGRAFÍA COMO UNA CONSTRUCCIÓN HISTÓRICA}

La presencia de la geografía escolar en Europa se rastrea según De Ávila y Ferias (2010) a principios siglo XIX, cuando Prusia configuraba el Estado-nación Alemán, el gobierno estableció la educación básica para todos, con la obligación de aprender el idioma nacional, la historia, y geografía desde la perspectiva del "amor a la patria." Adicionalmente, cargada con una función patriótica, la geografía también fue institucionalizada en Francia después de 1870 bajo la idea de una Alemania ganadora de la guerra Franco-Prusiana, a consecuencia de una tropa conocedora y amante de su patria. Tanto en Alemania y más tarde en Francia, la geografía se presenta, entonces, con un valor único al servicio del estado Estado-Nación. Sin embargo, hoy en ambas naciones según, Audigeir (1992) la geografía es valorada porque aporta una contribución esencial a la formación de los jóvenes, y les ayuda a convertirse en ciudadanos conscientes, activos y responsables.

La geografía como disciplina escolar en Colombia tiene una larga presencia en la escuela, los primeros atlas utilizados con una intención escolar provenían de ediciones españolas y norteamericanas, uno de ellos se denominó "Geografia general para el uso de las escuelas y familias arreglado para las repúblicas latinas de América", los primeros textos, narrativas de la existencia de un conocimiento geográfico escolar datan de 1825 en los albores de la independencia, un buen ejemplo es el libro de Pedro Acevedo Tejada, publicado en segunda edición en 1827. "Noticia sobre la geografía política de Colombia: proporcionada para la primera enseñanza de los niños en este importante ramo de la educación. Así mismo, las relaciones de la geografía escolar con la configuración del estado-nación para nuestro país, son estudiadas por Álvarez (2010) para las primeras décadas del siglo pasado.

Además las diferentes reformas educativas y de planes nacionales de educación en el siglo pasado contemplaron la geografía. Se podría afirmar que hasta el decreto 080 de 1974, reglamentario de los programas de secundaria, se comenzó a configurar un área de sociales que debía componerse de la Educación Ética, Moral y Religiosa, de la Filosofía, la Historia y la Geografía, en ese momento se denominó estudios sociales; con el decreto 1002 de 1984, oficialmente desaparece la denominación de geografía como asignatura escolar y se da la entrada al área de Ciencias Sociales. Originando así una mudanza, a más de un siglo, de denominación específica en geografía que había estado circulando y naturalizando la escuela. 
Por lo anterior, esa denominación de ciencias sociales en la escuela es más reciente que vieja, y aunque hoy este dándose su naturalización, es bueno considerar, además de los referentes históricos, algunas diferencias también espaciales que nos permiten observar que en otros lugares hoy, la geografía como disciplina escolar se mantiene, un buen ejemplo es Brasil ${ }^{4}$, país en el que solo se dio la integración de las ciencias sociales en la época de la dictadura; de cómo se dieron estos cambios y sus intencionalidades se encuentra en la investigación de Martins (2002) sobre la enseñanza de la historia escolar en el centro del debate (1971-1984). Diferentes estudios al respecto concluyeron que con la denominación de interdisciplinario se combinaron diferentes saberes, métodos, y contenidos claves de muy difícil articulación (De Ávila Melo, Farias y Freire 2010).

En México, Castañeda (2001), referencia que la geografía después de su conexión con las ciencias sociales, desde 1993 se enseñó de nuevo en las escuelas primarias y secundarias con una perspectiva apegada al constructivismo como método de enseñanza, además se incluyó la geografía en la formación de profesores de primaria en 1997 y la de profesores de geografía para secundaria, en 1999.

En general, los estudios referidos a la historia de las disciplinas escolares concuerdan con Goodson y Anstead (2003), que un análisis detallado de la estabilidad o cambio curricular de un saber escolar requiere una comprensión de su historia; recordemos que cualquier interpretación teórica, demanda una interpretación de los éxitos, fracasos e intencionalidades de este conocimiento en una relación biunívoca pasado - presente.

Igualmente para Goodson (1995) el currículo prescrito se compra como un testigo delas intenciones de la sociedad hacia el proceso de escolarización. A través de su una retórica propia, ámbito de las materias escolares, busca legitimar y justificar ciertas intenciones básicas de la escolarización.

La construcción de una didáctica especifica o general, de una disciplina escolar, no puede desconocer la dinámica propia y especifica de ese saber escolar; diferente al saber de la disciplina base, las disciplinas escolares son productos perfectamente diferenciados de las disciplinas científicas de referencia, que se rigen por reglas determinadas. Se encuentra así una cultura escolar, que Julia (1995) y Viñao (2002) parten de la concepción de la institución escolar no solo como producto de las cánones externos a ella, sino como una construcción social que produce para sí misma y transmite determinadas pautas culturales y creaciones propias.

Adicionalmente ninguna didáctica específica o general puede dejar de reconocer el papel de la disciplina escolar en la configuración de la disciplina base. Antes de la institucionalización de la geografía como una disciplina académica y científica en el país, quienes la debatieron y la escribieron fueron los profesores de la enseñanza media. Al respecto durante los años ' 80 han surgido múltiples investigadores (Capel, 1983; Gangas, 1985, Audigier 1992) que demostraron para España y Francia este proceso.

4 La asignatura Geografía enseñada en las escuelas comenzó a cambiar desde las profundidades creación de "Integración Social", que, en los programas escolares, en "Estudios Sociales". Resolución N 8, 1 de diciembre de 1971, el Consejo Federal de Educación, en virtud de la Ley de5692/71, que fijada a las enseñanzas mínimas comunes para los grados primero y segundo (la escuela primaria y Media), el establecimiento de los objetivos y la amplitud, lo que confirma que la Ley 4024/61 ha traído consigo La geografía como la integración social, más tarde llamada las Ciencias Sociales por la Resolución N ${ }^{\circ}$ 96/68. 


\section{INCERTIDUMBRES Y DINÁMICAS DE LA DIDÁCTICA GENERAL.}

La configuración de un campo de conocimiento en que se encuentran la didáctica de las ciencias sociales, como también la didáctica de la geografía, demanda un acercamiento a las particularidades y preocupaciones que configura la didáctica general, como un corpus tradicional, de flexiones y prácticas, que de una u otra manera vienen afianzado las mismas didácticas específicas, pero que también bebe de ellas para configurarse. Dentro de una amplia panorámica sobre lo que es la didáctica general, de su conjunto de conceptos complejos, de sus procesos, problemas y preguntas, enumeraremos las discusiones más cercanas a nuestra tradición así:

En primer, lugar se tiene hoy a la orden del día la propuesta didáctica de Klafki trabajada por Paredes (2011), cuya esencia fundamental está en aceptar que no se debe partir de supuestos teóricos sino de la realidad educativa para pensar tanto la enseñanza de los saberes como la formación de los sujetos; en términos generales, presenta Paredes, la manera en que los contenidos sean elegidos, deben atender tanto a su pertinencia para la comprensión del mundo del saber, como para la formación intelectual, actitudinal y práctica del sujeto. En últimas se está dejando a la discusión didáctica, el tema profundo de la formación.

Otras posturas rondan la idea de Camilloni (1993) sobre la discusión del carácter científico de la didáctica, que algunos la ubican en el marco de una concepción positivista o neopositivista de la ciencia. Para esta corriente, entonces, la didáctica es una disciplina que no puede ser considerada como un punto de vista científico porque está entrañablemente ligada a la búsqueda del logro práctico de los fines de la educación. Sin embargo, Aisenberg (2008) nos referencia cómo a través del conductismo y luego de los enfoques cognitivos y entre ellos, los que toman como eje la construcción de significados, plantean la urgencia de que la didáctica se constituya en un dominio del saber apoyado en el aporte riguroso de las disciplinas científicas.

Por otra parte, la discusión es aún más compleja ya que Camilloni (2004) postula como hipótesis que "el campo de la didáctica es reconocido y demarcado por los didactas, pero no es un campo reconocido por otras disciplinas" (p. 22), y al menos en didáctica de las ciencias sociales, ni siquiera es claro que todos los que trabajan en ella se asuman como didactas.

Por último, una postura que admite a la didáctica como un agregado de reflexiones apoyadas en diferentes ciencias, sin llegar a instituirse ella misma en una disciplina científica, por no contar con investigación específica ni con conceptos propios. Por lo que se ha intentado construir una didáctica inspirada en la psicología del aprendizaje (Aisenberg. 2008).

Desde otra óptica, se dan hoy discusiones de diversa magnitud sobre el análisis de las relaciones de los profesores con el saber didáctico, que Camilloni (1994) denomina saber erudito, y que implican una preocupación creciente por la formación didáctica de los profesores, tanto de los futuros docentes como también de los que están en ejercicio. Para esta postura se requiere producir cambios conceptuales, que permitan un proyecto y una práctica pedagógica. En últimas el propósito de la didáctica estaría en lograr que los estudiantes aprendan el saber erudito y no una versión manipulada y simplificada de ese saber.

Una de las conclusiones más relevantes de esta postura, encontrada en Camilloni (2005) es que las ideas sobre ser maestro y enseñar se forman muy tempranamente, asociadas con la construcción de 
la identidad, encontrándose en la irrupción de conceptos «básicos» que se desarrollan en la infancia, porque están referidos, en particular, a la asunción de los roles escolares. Es por ello entonces, que el cambio de las creencias debe efectuarse temprano en la formación, para que tenga resultados positivos.

Finalmente se encuentran dos posturas más amplias de la didáctica no conectadas directamente con la enseñanza: las reflexiones provenientes de la didáctica critica de Merchan (2006) más cercana a la historia de las disciplinas escolares; tiene como punto de partida otra definición de lo que es la escuela y de lo que son las prácticas escolares, que para este autor no están sólo en la cabeza de los profesores o en la mayor o menor capacidad de los alumnos, sino que contempla otras fuerzas, a veces invisibles, pero de una enorme potencia, y juegan un papel relevante, pues establecen las relaciones de unos y otros.

Igualmente las de Bolívar (1999) que defiende y postula una concepción ampliada de la didáctica, no restringida a cómo enseñar, sino que incluye los qué es y como es, en sus relaciones escuelasociedad. Esta visión de la Didáctica no tiene especial problema para asumir toda la tradición de investigación curricular. Podemos decir que hoy el alumno, dentro de las nuevas miradas de la didáctica, es un sujeto receptor, destinatario de la acción docente y ha pasado a ocupar un primer plano, donde los docentes representan el papel de mediadores (Sevillano 2004).

Finalmente abundan las críticas en relación a que la investigación didáctica, en general, ha estado más interesada en valorar cómo se enseña que lo que se enseña, aspecto que como señala Bolívar, (2005) le ha ganado un cierto rechazo por los profesores de las disciplinas, como los de secundaria. Pero sin duda, alguna la discusión más fuerte está del lado, de los que creen qué contenidos y didáctica, no se deben configurar en la formación del profesorado como campos separados.

\section{LA DIDÁCTICA DE LA GEOGRAFÍA: UN ESPACIO DE FUERTE CONFIGURACIÓN DISCIPLINAR.}

Nos referencia Bolívar (2005) que a mitad del XIX surge en el mundo alemán, la diferencia entre una didáctica general y una didáctica de las materias escolares, entendida ésta bajo los conceptos de "metodologías (Methodik)" y "didáctica especial". Bajo estas denominaciones se entendía que el concepto de didáctica especial, está implicando que la didáctica de las materias escolares debe desarrollarse con base a los fundamentos de la didáctica general. En la tradición alemana este contenido ha sido, tradicionalmente, uno de los elementos del triángulo didáctico (alumno, docente, contenido), cuyo tratamiento propio da lugar a las didácticas especiales.

Por otro lado, heredando la discusión de la didáctica general, las didácticas específicas, se preocuparon por pensar también como debían constituirse en disciplinas científicas, recurrieron a la investigación empírica sobre su propio objeto, retomaron los aportes de disciplinas relacionadas con la didáctica (como la psicología y las ciencias ligadas a los contenidos del área que se trate la enseñanza, entendida como una relación tríadica entre docente, alumnos y contenidos de un campo de conocimiento específico (2008, Aisenberg).

En general quienes trabajan como especialistas de didáctica en el área a la que pertenecen, han sido formados dentro de la disciplina, y articulan los distintos marcos teóricos de estas, junto a 
herramientas conceptuales pertinentes para analizar científicamente algún aspecto de la relación didáctica.

En la actualidad, a juicio de Calvo (2010), la didáctica de la geografía parece tener la necesidad de interrogarse acerca del momento epistemológico en que se encuentra su modelo de enseñanza, volcando toda su estructura conceptual y metodológica hacia una reflexión sobre la "educación para la ciudadanía". También para Pérez (2011) la situación del mundo, requiere que la geografía asuma los graves problemas que le afectan; es por ello que usando sus palabras se puede afirmar que la actual educación escolar no prepara a los alumnos para ser ciudadanos de nuestra sociedad, sino que responde a modelos tradicionales que se mantienen vigentes desde hace mucho tiempo, por lo que se demanda una educación ciudadana diferente.

Además de los temas claves que circulan dentro de la didáctica de la geografía que están articulados en los temas ambientales y de ciudadanía, han ido desplazando los problemas propios de las teorías cognitivas, que monopolizaron la disciplina en las décadas pasadas, referidas a la construcción del conceptos espaciales en niños y jóvenes; surgen una serie de preguntas entre las que destacan, las elaboradas por Calvo (2010), ¿qué tipo de relaciones habría que establecer entre la didáctica de la geografía con las nuevas tendencias de la investigación geográfica? Y en lo que se refiere a una enseñanza asociada a la formación para la práctica ¿cuál podría ser el estatus y el lugar de la didáctica en la formación de los profesores teniendo en cuenta los aportes teóricos de la geografía? (p.1).

En las últimas décadas, Calvo (2010), nos muestra el cambio de una geografía escolar con una fuerte tradición cimentada en las ciencias naturales, hacia una epistemología recentrada en las ciencias sociales y humanas, esto condujo a la modificación de los conocimientos de la geografía física que se enseña en secundaria, ahora estudiados en favor de problemáticas más generales (medio ambiente, degradación de la naturaleza, cambio climático), y que asocian a la geografía cada vez con más frecuencia, con cuestiones derivadas de la contaminación a nivel global y la sustentabilidad del planeta a medio y largo plazo.

Por otro lado, una dificultad de gran envergadura asumida por la didáctica de la geografía, tiende hoy a apabullarla; nos referimos a su estado de reconocimiento y legitimidad. Es decir, en la geografía escolar el problema suele estar en la geografía misma y para sostener una buena didáctica parece necesario ser un buen geógrafo ligado lo más cerca posible a la evolución de la comunidad pedagógica, de la que parte el fundamento epistemológico de toda didáctica, Calvo, (2010).

Finalmente, un nuevo objeto de interés está hoy presente dentro de la didáctica de la geografía, la aplicación de las nuevas tecnologías al quehacer diario del aula, pero también a la consolidación de un saber científico propio, a saber, el conocimiento geográfico e histórico educativos, González, (2002). Las clases de Geografía están como tantas otras disciplinas y áreas del conocimiento, impactadas por las tecnologías de la información y la comunicación.

En consecuencia, la didáctica de la geografía se configura como un campo autónomo con unas preguntas por resolver, con viejas y persistentes preocupaciones, sumadas a otras nuevas, vinculadas al uso didáctico de las tecnologías, a las reflexiones medio ambientales y de la ciudadanía, y a las reflexiones teóricas y metodológicas que aunque escasas, están definiéndola como una disciplina. 


\section{DE LA DIDÁCTICA DE LA GEOGRAFÍA A LA DIDÁCTICA DE LAS CIENCIAS SOCIALES: UN CAMPO EN CONTIENDA}

La didáctica general, las didácticas específicas de historia y geografía, fundamentalmente, con la didáctica de las ciencias sociales, estarían hoy configurando lo que Bourdieu,(1975) denominó como un campo. Los diversos agentes que participan en éste, las didácticas general, específicas y la pedagogía misma, son poseedores de un determinado capital cultural y de cierto tipo de habitus, tienen unas metodologías, cuerpos teóricos, preguntas y problemas, que las sitúa y les permiten detentar una posición, donde se disputan otras posiciones y otros capitales, que hacen del campo, un espacio social de fuerzas en constante lucha por la hegemonía de lo legítimo contra lo que se asume como ilegítimo y prohibido.

En este espacio de fuerzas se incluye no solo a los agentes sino también a las instituciones y sus sistemas de normas. Encontramos en este campo, unas fuerzas de tensión que se denominan ciencias sociales y que ejerce una fuerza de carácter centrípeta sobre la geografía y la historia para absorberles y configurarlas en una sola masa compacta, para ello se ha valido de las teorías de la interdisciplinaridad y de la legitimación del mundo escolar.

En el caso de la didáctica de la geografía, ésta se comenzó a institucionalizar en los años sesenta y setenta, gracias a las asociaciones de geógrafos, congresos y publicaciones, de esta época. La publicación clásica de ese momento se denominó La didáctica de la geografía: diez años de evolución de Patrick Baile en 1976; los conceptos y teorías eran discutidos, en las revistas Geography and the Teacher, Géography in Education, Heinemann, de 1976. Se suman a esta configuración los eventos y publicaciones del grupo de trabajo de didáctica de la Geografía de la Asociación de Geógrafos Españoles, adicionalmente el grupo de investigación de la enseñanza de la geografía de la UGI Unión Geográfica Internacional. Actualmente se configura en América Latina la Red Latinoamericana de Investigadores en Didáctica de la Geografía, que aglutina una variedad de investigadores y grupos a través de la revista Revista Virtual Anekumene además de una variedad de grupos de variada procedencia investigativa en Brasil que se agrupan en la Revista Brasileira de Educação em Geografia.

Por otra parte la Didáctica de las Ciencias Sociales, DCS, según Ávila (2010) se fue configurando desde el año 1984, mostrando su especial interés científico e investigador a través de la Asociación Universitaria del Profesorado de Didáctica de las Ciencias Sociales, que viene contribuyendo a la construcción de este campo de investigación específico con la celebración de simposios anuales, con foros de información, debate e intercambio de experiencias e investigadores. Programas de Doctorado y Máster en los Departamentos de Didácticas Específicas y la correspondiente lectura de tesis doctorales.

Para construir una didáctica de las ciencias sociales y establecer el carácter propio del conocimiento de sus contenidos es necesario según Camilloni,(1993) dar respuesta explícita a cuestiones tales como la determinación del status epistemológico de las ciencias sociales, de la misma manera, que las demás didácticas lo han hecho. Enfrentar la intricada dificultad de asumir las ciencias sociales, no como una integración de disciplinas, sino como una disciplina propia; ejercicio en el que se encuentra hoy, y en el que tiene que contar como lo hicieron las demás disciplinas en su momento,con la legitimización del mundo escolar. 
Y finalmente los problemas que enfrenta la didáctica de las ciencias sociales, están relacionados con las dificultades en la posibilidad de integración de las diferentes ciencias sociales entre sí, la indagación por el valor de la verdad o la certeza del conocimiento social, los límites de la objetividad o neutralidad de ese conocimiento y su relación con los valores y con la acción del hombre. Las preguntas en un primer nivel, son ¿qué características debe asumir la didáctica de las ciencias sociales como región disciplinar, en el marco de una disciplina mayor que la contiene, la didáctica general? Y, en segundo lugar y en un nivel más específico ¿qué enseñar y cómo enseñar en el dominio de las ciencias sociales? (Camilloni, 1993).

\section{A MANERA DE CONCLUSIÓN}

Los contenidos, las preguntas, y problemas de la enseñanza de las ciencias sociales provienen, en general, de campos disciplinarios organizados como la geografía, que están demarcados de acuerdo con los objetos del conocimiento de que se ocupan, con las metodologías de investigación y validación de los conocimientos que emplean, propias y de los temas que tratan con los conceptos que construyen. En tal sentido, se dan unas fuerzas de poder en términos de existencia, al configurarse una disciplina reciente como las ciencias sociales y otras de más vieja existencia, como la geografía, en donde la primera aspira a absorber la segunda.

En general la didáctica tanto la de las ciencias sociales, como de la geografía, son disciplinas teóricas, que se ocupan de estudiar el quehacer pedagógico, es decir, las prácticas de la enseñanza, y que tienen como misión describirlas, explicar, fundamentar y enunciar normas para la mejor resolución de los problemas, que estas prácticas plantean a los profesores. Sin embargo, actualmente las teorías sicológicas están cambiando el objeto de la didáctica desde la enseñanza para girar en función del aprendizaje, este hecho ha invadido con mayor fuerza las didácticas específicas, y están deslocalizando al maestro colocándolo como un dinamizador.

\section{REFERENCIAS}

Aisenberg B. (2003). Didáctica de las ciencias sociales: ¿Desde qué teoría Estudiamos la enseñanza? 2003 Aisenberg.En: http://www.saber.ula.ve/handle/123456789/23916 Acceso 8 de mayo 2012

Bolívar A. (2005) Conocimiento didáctico del contenido y didácticas específicas www.ugr.es/ recfpro/ rev92ART6.pdf.

Bourdieu, P. (1975).Campo del poder y campo intelectual. Argentina: Folios.

Camillon A. (sf) Epistemología de la didáctica de las ciencias sociales En:http://rubenama.com/articulos/camilloni_epistemologia_didactica_ccss.pdf Acceso 16 de marzo 2012.

(2005) Formación de Profesores y Transposición Didáctica http://132.248.9.1:8991/ hevila/pdf-| ariel/Foro\%20Educacional\%20No.\%207,\%202a.\%20epoca,\%201er.\%20 seme stre\%202005.pdf 
.(1998). El sujeto del discurso didáctico. En Praxis Educativa. Revista del Instituto de Ciencias de la Educación para la Investigación Interdisciplinaria. Universidad Nacional de La Pampa. Año III, Nº3.

Castañeda, R. (2001).La geografia escolar en México: 1821-2000,En| http://www.inegi.org.mx/inegi/ contenidos/espanol/prensa/contenidos/articulossociodemograficas/geografia03.pdf. Acceso:6 de junio 2012 .

Díaz, M. (1993) El campo intelectual de la educación en Colombia, Cali, Universidad del Valle, 1993

Franqois A. (1992). Pensar la geografia escolar. Un repte per a la didàctica. Documents d'Anàlisi Geogràfica, $n^{\circ}$ 21, Bellaterra (Barcelona), 1992, p. 15-3

Garcés G. (2002). El campo conceptual de la pedagogía, aproximaciones a un debate necesario. En: isabelreynel.wikispaces.com/.../El+campo+conceptual+de+la+pedagogía.... Acceso 21 abril 2012

García, P. (2011). Problemas del mundo y educación escolar. En Geografía, Problemas Sociales Y conocimiento escolar. En www.anekumene.com/index.php/revista/article/download/24/2, Acceso 21 abril 2012

Goodson, F. \& Anstead, C.(2003). Detrás de las puertas de la escuela: el Estudio histórico del curriculum. GOODSON, Ivor F. (coord.). Estudio del curriculum: casos y métodos. Buenos Aires: Amorrortu

Melo, A., De Avila; Vlac Vania R. (Sf) Historia da geografia escolar Brasileira: En http://www.faced.ufu.br/colubhe06/anais/arquivos/239AdrianyMelo VaniaRub ia.pdf. Acceso 23 de julio 2012

Merchán, J. (2006) Crítica de la didáctica: conocimiento, formación del profesorado y mejora de la enseñanza profesorado y mejora de la enseñanza. En: www.fedicaria.org/miembros/nebraska/3.pdf,Acceso 4 de julio 2012

González, M. (2005). Nuevas tecnologías aplicadas a la didáctica de la geografia: el ejemplo de google earth En: Zaragozarafaeldemiguel.files.wordpress.com/.../nuevas-tecnologiasacceso 6 de marzo 2012.

Paredes, D. (2011). Pensar la enseñanza de la filosofia desde la didáctica teórico-formativa. En:virtual.uptc. edu.co/revistas/index.php/cuestiones_filosofia/.../806 Formato de archivo: PDF/Adobe Acrobat - Vista rápidae DM Acceso 23 de julio 2012.

Sevillano García (2004,) Didáctica y currículum; Controversia Inacabada. Disponible en: dialnet.unirioja.es/servlet/articulo?codigo=122417 www.fedicaria.org/miembros/nebraska/3.pdf, Acceso 23 de julio 2012.

Articulo recibido en abril 5 de 2013 - Aprobado en mayo 15 de 2013 